\title{
Form-factors of the sausage model obtained with bootstrap fusion from sine-Gordon theory
}

\author{
Zalán Horváth \\ Institute for Theoretical Physics \\ Roland Eötvös University, Budapest, Hungary \\ Gábor Takács \\ Department of Applied Mathematics and Theoretical Physics \\ Cambridge, UK \\ (6 November 1995)
}

\begin{abstract}
We continue the investigation of massive integrable models by means of the bootstrap fusion procedure, started in our previous work on $\mathrm{O}(3)$ nonlinear sigma model. Using the analogy with $\mathrm{SU}(2)$ Thirring model and the $\mathrm{O}(3)$ nonlinear sigma model we prove a similar relation between sine-Gordon theory and a one-parameter deformation of the $\mathrm{O}(3)$ sigma model, the sausage model. This allows us to write down a free field representation for the Zamolodchikov-Faddeev algebra of the sausage model and to construct an integral representation for the generating functions of formfactors in this theory. We also clear up the origin of the singularities in the bootstrap construction and the reason for the problem with the kinematical poles.
\end{abstract}

\section{INTRODUCTION}

Exactly solvable two-dimensional quantum field theories have attracted a great deal of interest and research work recently. There are several reasons for that: these theories can serve as laboratory for higher dimensional theories; there are physical systems with effectively two-dimensional phenomena (eg. quantum Hall effect); and there is the huge and rapidly developing area of string theory, topological field theory and related topics, in which two-dimensional physics plays a central role.

A large variety of different approaches emerged in trying to solve these models of quantum field theory. The first real breakthrough was achieved in [2], in which exact S-matrices were conjectured for several interesting models using bootstrap ideas. The fact that made this possible is that scattering theory of quantum integrable models is very simple: it is factorized scattering theory (FST). This means that the many-particle S-matrix can be written in terms of products of two-particle S-matrices. The consistency condition for this turns out to be the famous Yang-Baxter equation, which, together with unitarity, crossing symmetry and the spectrum of the particles of the model, severely restricts the class of possible S-matrices that one can write down. However, there is a so-called CDD ambiguity, which means that the S-matrix is not determined uniquely by the aforementioned conditions. In most cases one solves this difficulty by choosing a 'minimal' solution to the factorization equations. This choice can be justified in many cases by comparison of results coming from the conjectured S-matrix and from the Lagrangian field theory.

The S-matrix, however, describes only the on-shell physics. For off-shell physics, one turns to other quantities which contain more information: correlation functions. For their calculation the knowledge of the matrix elements of local operator is needed. These matrix elements are called form-factors; correlation functions can be represented as an infinite series constructed using these objects. For the majority of integrable field theories this is the only tool to compute correlation functions up to now; in some simple cases one can use other techniques.

Form-factors can be found solving a very complicated system of axioms, which have been known for a long time [3]. The two-particle minimal form-factors were calculated for many models, however the calculation of the many-particle ones posed essential difficulties. Then, in a remarkable series of papers, Smirnov [4] obtained all form-factors of some important operators in the sine-Gordon, $\mathrm{SU}(2)$ Thirring and $\mathrm{O}(3)$ sigma models. Further investigation revealed that the key structure behind these solutions is that of quantum affine algebras, which is a nonlocal symmetry of these integrable field theories [8]. The Zamolodchikov-Faddeev operators (which play a central role in our approach as well) turned out to be vertex operators for the representations of the nonlocal symmetry algebra [9].

The analogy between the Knizhnik-Zamolodchikov (KZ) equations for affine Kac-Moody algebras and the formfactor axioms, one of which proved to be a quantum analogue of the $\mathrm{KZ}$ equation [9, 13], as well as the vertex operator representation of quantum affine algebras 14 strongly suggest that a bosonization technique based on free fields, similar to the one used in conformal field theory, should be useful in solving the form-factor equations. This is the central idea in the papers of Lukyanov [15,16], where he was able to construct a free field representation for the sineGordon and SU(2) Thirring model and to obtain an integral representation for the form factors. In our earlier work [1], using mainly his results and the connection between $\mathrm{SU}(2)$ Thirring and $\mathrm{O}(3)$ sigma models put forward by Smirnov, we defined a free field representation for the $\mathrm{O}(3)$ model and applied it to build up an integral representation for the 
form-factors. Here we extend the method to the sine-Gordon and sausage models and in this way write down a similar representation for the form-factors of the sausage model. The problems encountered in [1] when applying the method unfortunately remain the same and we choose the same old solution for correcting the analytic structure introducing some multiplier functions. Our main result is to obtain a procedure following which one can calculate form-factors of the sausage model. We also provide a detailed analysis of the analytical structure and an interpretation of the double poles in terms of an analogy with thermal Green functions. The relation of the problem of the kinematical poles and the singularity of the fusion limit of the sine-Gordon form-factor is cleared up.

The paper is organized as follows. Section II reviews factorized scattering theory of sine-Gordon and sausage models and there we discuss the quantum symmetries. In Section III we briefly describe the form-factor axioms and the free field representation for their solution. Section IV deals with the question of the bootstrap fusion procedure used for transition from the sine-Gordon model to the sausage model and using these results defines the free field representation for the sausage model, while in Section $\mathrm{V}$ we give the general formulas for evaluating the form-factors and provide a detailed discussion of their analytic structure. Section VI is reserved for the conclusions.

We added three appendices to the paper. Appendix A describes the method used to obtain the bootstrap fusion and the Zamolodchikov-Faddeev algebra relation on the level of the free field representation, while Appendix B shows how to evaulate the traces occurring in the calculation of generating functions with coherent oscillator methods. In Appendix $\mathrm{C}$ we describe a sample calculation of the operator product singularities.

\section{REVIEW OF THE SINE-GORDON AND SAUSAGE FIELD THEORIES}

Sine-Gordon theory is defined by the Lagrangian density

$$
\mathcal{L}=\frac{1}{2}\left(\partial_{\mu} \phi\right)^{2}+\frac{m_{0}^{2}}{b^{2}} \cos (b \phi),
$$

where $b$ is a coupling constant and $m_{0}$ is a mass parameter. Instead of $b$ we will use the parameter

$$
\xi=\frac{b^{2}}{8 \pi-b^{2}},
$$

which can be taken as the only dimensionless parameter of the model. When $\xi>1$, the quantum spectrum of the theory contains only a doublet of solitons which forms the fundamental representation of the quantum group $S U(2)_{q}$ with

$$
q=\exp \left[i \pi\left(1+\frac{1}{\xi}\right)\right] .
$$

When $\xi<1$, the spectrum contains breathers, which are bound states of the fundamental solitons. We do not treat this case here. We also restrict ourselves to study the case when $q$ is not a root of unity to avoid dealing with degenerate representations of the quantum group. The S-matrix of the solitons was found in [2] and looks as follows:

$$
\begin{aligned}
& S_{++}^{++}(\beta)=S_{--}^{--}(\beta)=S_{0}(\beta), \\
& S_{+-}^{+-}(\beta)=S_{-+}^{-+}(\beta)=S_{0}(\beta) \frac{\sinh \left(\frac{\beta}{\xi}\right)}{\sinh \left(\frac{i \pi-\beta}{\xi}\right)}, \\
& S_{-+}^{+-}(\beta)=S_{+-}^{-+}(\beta)=S_{0}(\beta) \frac{\sinh \left(\frac{i \pi}{\xi}\right)}{\sinh \left(\frac{i \pi-\beta}{\xi}\right)},
\end{aligned}
$$

where,+- is the internal index, $\beta$ is the rapidity parameter, which is related to the energy-momentum by $p_{0}=$ $m \cosh \beta, p_{1}=m \sinh \beta, \mathrm{m}$ is the soliton mass and

$$
S_{0}(\beta)=-\exp \left[-i \int_{0}^{\infty} \frac{\sin (\kappa \beta) \sinh \left(\frac{1-\xi}{2} \pi \kappa\right) d \kappa}{\kappa \cosh \left(\frac{1}{2} \pi \kappa\right) \sinh \left(\frac{\xi}{2} \pi \kappa\right)}\right] .
$$

The Hilbert space of the theory can be described by introducing the formal Zamolodchikov-Faddeev operators $Z_{i}(\beta)$ satisfying the following algebra relations 


$$
Z_{i}\left(\beta_{1}\right) Z_{j}\left(\beta_{2}\right)=S_{i j}^{k l}\left(\beta_{12}\right) Z_{l}\left(\beta_{2}\right) Z_{k}\left(\beta_{1}\right), \quad \beta_{12}=\beta_{1}-\beta_{2}
$$

As we shall see below, the commutation relations of the Z-operators reflect the scattering of the particles. From now on we will refer to (2.6) as the scattering relations for the sine-Gordon model. The Hilbert space furnishes a representation of the Zamolodchikov-Faddeev (ZF) algebra. The ZF operators create the asymptotic in and out states:

$$
\begin{aligned}
& \left|Z_{i_{1}}\left(\beta_{1}\right) \ldots Z_{i_{n}}\left(\beta_{n}\right)\right\rangle_{\text {out }}=Z_{i_{1}}\left(\beta_{1}\right) \ldots Z_{i_{n}}\left(\beta_{n}\right)|\mathrm{vac}\rangle, \\
& \left|Z_{i_{1}}\left(\beta_{1}\right) \ldots Z_{i_{n}}\left(\beta_{n}\right)\right\rangle_{\text {in }}=Z_{i_{n}}\left(\beta_{n}\right) \ldots Z_{i_{1}}\left(\beta_{1}\right)|\mathrm{vac}\rangle,
\end{aligned}
$$

where $\mid$ vac $\rangle$ is the vacuum state and the rapidities are ordered as $\beta_{1}<\beta_{2}<\ldots<\beta_{n}$. Now if one expresses the out states in terms of the in states, one gets the $n$-particle S-matrix as a product of the 2 -particle S-matrices. The associativity of the ZF algebra then implies the famous Yang-Baxter equation, which is the factorization condition for the S-matrix [2].

The Hilbert space structure is specified by defining the conjugate ZF operators $Z^{i \dagger}$, which annihilate the vacuum and satisfy the following additional relations

$$
\begin{aligned}
& Z^{i \dagger}\left(\beta_{1}\right) Z^{j \dagger}\left(\beta_{2}\right)=Z^{l \dagger}\left(\beta_{2}\right) Z^{k \dagger}\left(\beta_{1}\right) S_{k l}^{i j}\left(\beta_{12}\right), \\
& Z_{i}\left(\beta_{1}\right) Z^{j \dagger}\left(\beta_{2}\right)=Z^{k \dagger}\left(\beta_{2}\right) S_{i k}^{j l}\left(\beta_{12}\right) Z_{l}\left(\beta_{1}\right)+2 \pi \delta_{i}^{j} \delta\left(\beta_{12}\right) .
\end{aligned}
$$

However, in the sequel we will deal with another representation of the ZF algebra defined by (2.6) which does not include the conjugate ZF operators and therefore it is different from the physical Hilbert space of the model. This representation will be the space of the free field system used to solve the form-factor equations.

As mentioned above, the sine-Gordon model is invariant under $S U(2)_{q}$ quantum group symmetry. To make this symmetry manifest, one has to redefine the ZF operators

$$
\hat{Z}_{a}(\beta)=\exp \left(a \frac{\beta}{2 \xi}\right) Z_{a}(\beta),
$$

which implies the following redefinition of the S-matrix

$$
\hat{S}_{a b}^{c d}(\beta)=\exp \left((a-c) \frac{\beta}{2 \xi}\right) S_{a b}^{c d}(\beta) .
$$

It can be easily proved that if we identify the vectors $\hat{Z}_{a}(\beta) \mid$ vac $\rangle$ as the fundamental representation of the quantum group $S U(2)_{q}$, then the redefined S-matrix and the scattering relations (2.6) are invariant under the action of the quantum group. However, care must be taken since $\hat{S}$ is not a unitary matrix and the representation of the out states differs from that of the in states by a change $q \rightarrow 1 / q$. Since $|q|=1$, this is equivalent to a complex conjugation. Using this invariance and the fact that the decomposition of the corresponding representations is given by $\frac{1}{2} \otimes \frac{1}{2}=0 \oplus 1$ (we label the representations using the conventional isospin notation), we can extract the amplitudes in the two possible channels which turn out to be

$$
S_{\text {isospin }-1}(\beta)=S_{0}(\beta), S_{\text {isospin-0 }}(\beta)=\frac{\sinh \left(\frac{i \pi-\beta}{\xi}\right)}{\sinh \left(\frac{i \pi+\beta}{\xi}\right)} S_{0}(\beta),
$$

and $\hat{S}$ can be decomposed as

$$
\hat{S}=S_{\text {isospin-0 }} P_{0}+S_{\text {isospin-1 }} P_{1},
$$

where $P_{0}$ and $P_{1}$ are projectors on the spin- 0 and spin- 1 subspace of the product of two spin- $1 / 2$ representations.

The sausage model was introduced in $\sqrt{17}$ as a one-parameter family of deformations of the $\mathrm{O}(3)$ nonlinear sigma model. The spectrum of the model consists of a massive (quantum group) isotriplet of scalars, capital letters $I, J, K, L$ will be used to label the internal quantum number which takes the values $+, 0,-$. The S-matrix is invariant under a $U(1)$ symmetry and the charge conjugation which takes $\bar{I}=-I$. The S-matrix has the following symmetries:

$$
\begin{aligned}
& \mathcal{S}_{I J}^{K L}(\beta)=\mathcal{S}_{\bar{I} \bar{J}}^{\bar{K}} \bar{L}(\beta)=\mathcal{S}_{J I}^{L K}(\beta)=\mathcal{S}_{K L}^{I J}(\beta) \quad(\mathrm{C}, \mathrm{P}, \mathrm{T}), \\
& \mathcal{S}_{I J}^{K L}(\beta)=\mathcal{S}_{J \bar{I}}^{L \bar{K}}(i \pi-\beta) \quad \text { (crossing) . }
\end{aligned}
$$


The independent S-matrix-elements are

$$
\begin{aligned}
& \mathcal{S}_{++}^{++}(\beta)=\frac{\sinh \lambda(\beta-i \pi)}{\sinh \lambda(\beta+i \pi)}, \\
& \mathcal{S}_{+0}^{0+}(\beta)=-i \frac{\sin 2 \pi \lambda}{\sinh \lambda(\beta-2 i \pi)} \mathcal{S}_{++}^{++}(\beta), \\
& \mathcal{S}_{+-}^{-+}(\beta)=-\frac{\sin \pi \lambda \sin 2 \pi \lambda}{\sinh \lambda(\beta+i \pi) \sinh \lambda(\beta-2 i \pi)}, \\
& \mathcal{S}_{+0}^{+0}(\beta)=\frac{\sinh \lambda \beta}{\sinh \lambda(\beta-2 i \pi)} \mathcal{S}_{++}^{++}(\beta), \\
& \mathcal{S}_{00}^{00}(\beta)=\mathcal{S}_{+0}^{+0}(\beta)+\mathcal{S}_{+-}^{-+}(\beta),
\end{aligned}
$$

where $\lambda$ is a parameter of the model. This is a consistent S-matrix if $0 \leq \lambda \leq 1 / 2$; otherwise, there are poles inside the physical sheet $0 \leq \operatorname{Im} \beta<\pi$. In [17] the one-loop renormalized action can be found. For our considerations we will need only the S-matrix (2.14).

The S-matrix of the sausage-model is $S U(2)_{q}$ invariant with $q=\exp [i \pi(\lambda+1)]$. When we set $\lambda \rightarrow 0$, we find the S-matrix of the $\mathrm{O}(3)$ nonlinear sigma model. One can introduce the ZF operators $\mathcal{Z}_{I}(\beta)$ for the sausage model similarly to the case of sine-Gordon theory. The basis needed to exhibit the quantum group symmetry is given by

$$
\hat{\mathcal{Z}}_{I}(\beta)=\exp (\lambda I \beta) \mathcal{Z}_{I}(\beta) .
$$

The states created by the operators $\hat{\mathcal{Z}}$ form a triplet (isospin-1) representation of $S U(2)_{q}$. Similarly to the case of sine-Gordon model (see (2.12), we can decompose the S-matrix into three channels corresponding to $1 \otimes 1=0 \oplus 1 \oplus 2$. The amplitudes for the three channels are

$$
\begin{aligned}
& \mathcal{S}_{0}(\beta)=\frac{\sinh \lambda(\beta+2 i \pi)}{\sinh \lambda(\beta-2 i \pi)} \\
& \mathcal{S}_{1}(\beta)=\frac{\sinh \lambda(\beta+2 i \pi)}{\sinh \lambda(\beta-2 i \pi)} \frac{\sinh \lambda(\beta-i \pi)}{\sinh \lambda(\beta+i \pi)} \\
& \mathcal{S}_{2}(\beta)=\frac{\sinh \lambda(\beta-i \pi)}{\sinh \lambda(\beta+i \pi)}
\end{aligned}
$$

\section{FORM-FACTOR AXIOMS AND THE FREE FIELD REPRESENTATION FOR THEIR SOLUTION}

In Smirnov's approach to integrable field theories, the local operators are defined in terms of their form-factors. Form-factors are the matrix elements of the local operators between the vacuum and many-particle states:

$$
F_{i_{1}, \ldots, i_{n}}^{\mathcal{O}}\left(\beta_{1}, \ldots, \beta_{n}\right)=\left\langle\operatorname{vac}|\mathcal{O}(0)| \beta_{1}, \ldots, \beta_{n}\right\rangle_{i_{1}, \ldots i_{n}} .
$$

By virtue of crossing symmetry, these matrix elements determine the general matrix element between two manyparticle states and, assuming asynptotic completeness of Hilbert space, this means the complete characterization of the local operator $\mathcal{O}(x)$. The form-factors of a local operator must satisfy the following set of axioms:

1. The functions $F$ are analytic in the rapidity differences $\beta_{i j}=\beta_{i}-\beta_{j}$ inside the strip $0<\operatorname{Im} \beta<2 \pi$ except for simple poles. The physical matrix elements are the values of the $F$ 's at real rapidity arguments ordered as $\beta_{1}<\beta_{2}<\ldots<\beta_{n}$.

2. The form-factor of a local operator of Lorentz spin $s$ satisfies

$$
F_{i_{1}, \ldots, i_{n}}\left(\beta_{1}+\Lambda, \ldots, \beta_{n}+\Lambda\right)=\exp (s \Lambda) F_{i_{1}, \ldots, i_{n}}\left(\beta_{1}, \ldots, \beta_{n}\right) .
$$

This means that the form-factor of a spin $s=0$ operator depends only on the rapidity differences.

3. Form-factors satisfy Watson's symmetry property:

$$
\begin{aligned}
& F_{i_{1}, \ldots, i_{j}, i_{j+1}, \ldots, i_{n}}\left(\beta_{1}, \ldots, \beta_{j}, \beta_{j+1}, \ldots, \beta_{n}\right)= \\
& S_{i_{j} i_{j+1}}^{k_{j+1} k_{j}} F_{i_{1}, \ldots, k_{j+1}, k_{j}, \ldots, i_{n}}\left(\beta_{1}, \ldots, \beta_{j+1}, \beta_{j}, \ldots, \beta_{n}\right) .
\end{aligned}
$$


4. Form-factors satisfy the cyclic property

$$
F_{i_{1}, \ldots, i_{n}}\left(\beta_{1}, \ldots, \beta_{n}+2 \pi i\right)=F_{i_{n}, i_{1}, \ldots, i_{n-1}}\left(\beta_{n}, \beta_{1}, \ldots, \beta_{n-1}\right) .
$$

In the case of an operator which has a mutual locality index $\omega(\mathcal{O}, \Psi)$ with respect to the elementary field $\Psi$, this axiom is modified to

$$
F_{i_{1}, \ldots, i_{n}}\left(\beta_{1}, \ldots, \beta_{n}+2 \pi i\right)=\exp [2 \pi i \omega(\mathcal{O}, \Psi)] F_{i_{n}, i_{1}, \ldots, i_{n-1}}\left(\beta_{n}, \beta_{1}, \ldots, \beta_{n-1}\right) .
$$

5. Form-factors have kinematical singularities which are simple poles at points where two of the rapidity arguments are displaced by $i \pi$. The residue is given by the following formula:

$$
\begin{aligned}
& \operatorname{res}_{\beta_{i}=\beta_{n}+i \pi} F_{i_{1}, \ldots, i_{n}}\left(\beta_{1}, \ldots, \beta_{n}\right)=-i C_{i_{n}, i_{j}^{\prime}} F_{i_{1}^{\prime}, \ldots, \hat{i}_{j}^{\prime} \ldots, i_{n-1}^{\prime},}\left(\beta_{1}, \ldots, \hat{\beta}_{j}, \ldots, \beta_{n}\right) \times \\
& {\left[\delta_{i_{1}}^{i_{1}^{\prime}} \ldots \delta_{i_{j-1}}^{i_{j-1}^{\prime}} S_{i_{n-1} k_{1}}^{i_{n-1} i_{j}^{\prime}}\left(\beta_{n-1}-\beta_{j}\right) S_{i_{n-2} k_{2}}^{i_{n-2}^{\prime} k_{1}}\left(\beta_{n-2}-\beta_{j}\right) \ldots S_{i_{j+1} i_{j}}^{i_{j+1}^{\prime} k_{n-j}}\left(\beta_{j+1}-\beta_{j}\right)\right.} \\
& \left.-S_{k_{1}, i_{1}}^{i_{j}^{\prime} i_{1}^{\prime}}\left(\beta_{j}-\beta_{1}\right) \ldots S_{k_{j-2} i_{j-2}}^{k_{j-3} i_{j-2}^{\prime}}\left(\beta_{j}-\beta_{j-2}\right) S_{i_{j} i_{j-1}}^{k_{j-2} i_{j-1}^{\prime}}\left(\beta_{j}-\beta_{j-1}\right) \delta_{i_{j+1}}^{i_{j+1}} \ldots \delta_{i_{n-1}}^{i_{n-1}^{\prime}}\right] .
\end{aligned}
$$

We would like to remark here that the cyclicity axiom closely resembles the KMS condition for finite-temperature Green functions of quantum field theory. We will see that the expressions for the solutions of the form-factor equations really look like expectation values in a heat bath and later we will use this analogy.

The locality theorem proved in [7] ensures that the operators defined by solutions of the above equations are local (up to the mutual locality indices, if there are such). The importance of the form-factors is given by the fact that the correlation function of two local operators can be expanded into an infinite series in terms of their form-factors, obtained by inserting a complete set of many-particle states into the correlator.

In the paper of Lukyanov [16], a free field representation for the Zamolodchikov-Faddeev algebra was proposed to solve the above axioms. That work considered the SU(2) Thirring and the sine-Gordon models. Here we recall some essential facts about this free field realization. We do not give a full treatment and restrict ourselves to the formulas, used in describing sine-Gordon form-factors, which are necessary in the subsequent sections. The interested reader is referred to [16] [18] for more details.

Let us introduce a field $\phi(\beta)$ with the following properties:

$$
\begin{aligned}
& {\left[\phi\left(\beta_{1}\right), \phi\left(\beta_{2}\right)\right]=\ln S_{0}\left(\beta_{2}-\beta_{1}\right),} \\
& \left\langle 0\left|\phi\left(\beta_{1}\right) \phi\left(\beta_{2}\right)\right| 0\right\rangle=-\ln g\left(\beta_{2}-\beta_{1}\right),
\end{aligned}
$$

where $S_{0}$ is the function defined in eqn. (2.5) and we give the formula for the function $g$ below (3.11). The consistency of the two relations above requires

$$
S_{0}(\beta)=\frac{g(-\beta)}{g(\beta)} .
$$

The field $\phi$ is represented (after a proper ultraviolet cut-off procedure) on a Fock space. We will build up operators which represent the ZF algebra on that space.

The field

$$
\bar{\phi}(\beta)=\phi\left(\beta+i \frac{\pi}{2}\right)+\phi\left(\beta-i \frac{\pi}{2}\right)
$$

will be important to define the screening charge below. Its two-point functions can be written down as

$$
\begin{aligned}
& \left\langle 0\left|\bar{\phi}\left(\beta_{1}\right) \phi\left(\beta_{2}\right)\right| 0\right\rangle=\ln w\left(\beta_{2}-\beta_{1}\right), \\
& \left\langle 0\left|\bar{\phi}\left(\beta_{1}\right) \bar{\phi}\left(\beta_{2}\right)\right| 0\right\rangle=-\ln \bar{g}\left(\beta_{2}-\beta_{1}\right),
\end{aligned}
$$

where we defined the following functions

$$
\begin{aligned}
& g(\beta)=\left(\frac{\kappa}{\Gamma\left(\frac{1}{\xi}\right)}\right)^{\frac{1}{2}} \frac{\Gamma\left(\frac{1}{\xi}+\frac{i \beta}{\pi \xi}\right)}{\Gamma\left(\frac{i \beta}{\pi \xi}\right)} \prod_{l=1}^{\infty} \frac{\left[R_{l}(i \pi) R_{l}(0)\right]^{\frac{1}{2}}}{R_{l}(\beta)} \\
& w(\beta)=\kappa^{-1} \frac{\Gamma\left(-\frac{1}{2 \xi}+\frac{i \beta}{\pi \xi}\right)}{\Gamma\left(1+\frac{1}{2 \xi}+\frac{i \beta}{\pi \xi}\right)}, \\
& \bar{g}(\beta)=\kappa^{2} \frac{i \beta}{\pi \xi} \frac{\Gamma\left(1+\frac{1}{\xi}+\frac{i \beta}{\pi \xi}\right)}{\Gamma\left(-\frac{1}{\xi}+\frac{i \beta}{\pi \xi}\right)}
\end{aligned}
$$


where $k$ is a normalization constant and $R_{l}$ is the function

$$
R_{l}(\beta)=\frac{\Gamma\left(\frac{2 l}{\xi}+\frac{i \beta}{\pi \xi}\right) \Gamma\left(1+\frac{2 l}{\xi}+\frac{i \beta}{\pi \xi}\right)}{\Gamma\left(\frac{2 l+1}{\xi}+\frac{i \beta}{\pi \xi}\right) \Gamma\left(1+\frac{2 l-1}{\xi}+\frac{i \beta}{\pi \xi}\right)} .
$$

Now we define Lukyanov's vertex operators:

$$
\begin{aligned}
& V(\beta)=\exp (i \phi(\beta))=(g(0))^{\frac{1}{2}}: \exp (i \phi(\beta)):, \\
& \bar{V}(\beta)=\exp (-i \bar{\phi}(\beta))=(\bar{g}(0))^{\frac{1}{2}}: \exp (-i \bar{\phi}(\beta)):,
\end{aligned}
$$

where : denotes an appropriate normal ordering [16]. These operators need to be regularized because the functions $g$ and $\bar{g}$ have simple zeros at $\beta=0$. Using the analogy with conformal field theory we define the regularized values of the functions $g, \bar{g}$ at $\beta=0$ to be

$$
\begin{aligned}
& g_{r e g}(0)=\lim _{\beta \rightarrow 0} \frac{g(\beta)}{\beta}=: \rho^{2}, \\
& \bar{g}_{r e g}(0)=\lim _{\beta \rightarrow 0} \frac{\bar{g}(\beta)}{\beta}=: \bar{\rho}^{2} .
\end{aligned}
$$

The vertex operators defined in (3.13) obey the following fundamental relations:

$$
\begin{aligned}
& V\left(\beta_{1}\right) V\left(\beta_{2}\right)=\rho^{2} g\left(\beta_{2}-\beta_{1}\right): V\left(\beta_{1}\right) V\left(\beta_{2}\right):, \\
& \bar{V}\left(\beta_{1}\right) V\left(\beta_{2}\right)=\rho \bar{\rho} w\left(\beta_{2}-\beta_{1}\right): V\left(\beta_{1}\right) V\left(\beta_{2}\right):, \\
& \bar{V}\left(\beta_{1}\right) \bar{V}\left(\beta_{2}\right)=\bar{\rho}^{2} \bar{g}\left(\beta_{2}-\beta_{1}\right): \bar{V}\left(\beta_{1}\right) \bar{V}\left(\beta_{2}\right): .
\end{aligned}
$$

Eqn. (3.15) plays a key role in proving the ZF scattering relations.

Then in analogy with the Coulomb gas representation of rational conformal field theory one introduces a screening charge $\chi$ by the definition

$$
\langle u|\chi| v\rangle=\eta^{-1}\left\langle u\left|\frac{1}{2 \pi} \int_{C} d \gamma \bar{V}(\gamma)\right| v\right\rangle,
$$

where $\mathrm{C}$ is a contour specified in the following manner: assuming that all matrix elements $\langle u|\bar{V}(\gamma)| v\rangle$ are meromorphic functions decreasing at the infinity faster than $\gamma^{-1}$, the contour goes from $\Re \gamma=-\infty$ to $\Re \gamma=+\infty$ and lies above all singularities whose positions depend on $|u\rangle$ and below all singularities depending on $|v\rangle$. ( $\eta$ is a normalization parameter which is chosen to get the correct residue for the pole on the operator product below (see [16])).

Having defined these objects, one can write down the following operators:

$$
\begin{aligned}
& Z_{+}(\beta)=\exp \left(-\frac{\beta}{2 \xi}\right) V(\beta), \\
& Z_{-}(\beta)=\exp \left(\frac{\beta}{2 \xi}\right)\left(q^{\frac{1}{2}} \chi V(\beta)-q^{-\frac{1}{2}} V(\beta) \chi\right) .
\end{aligned}
$$

Then the following propositions hold 16:

(i) These operators satisfy the $\mathrm{SU}(2)$ Thirring model ZF relations.

(ii) The singular part of the operator product $Z_{i}\left(\beta_{2}\right) Z_{j}\left(\beta_{1}\right)$ considered as a function of the complex variable $\beta_{2}$ for real $\beta_{1}$ in the upper half plane $\Im \beta_{2} \geq 0$ contains only one simple pole at $\beta_{2}=\beta_{1}+i \pi$ with residue $-i C_{i j}$.

We introduce the following structures, specified later in details:

1. The operator of Lorentz boosts $K$ satisfying

$$
Z_{i}(\beta+\Lambda)=\exp (-\Lambda K) Z_{i}(\beta) \exp (\Lambda K)
$$

2. A map $\mathcal{O} \rightarrow L(\mathcal{O})$ from the space of local operators to the algebra of endomorphisms of $\pi_{Z}$ satisfying the following two conditions

$$
\begin{aligned}
L(\mathcal{O}) Z_{i}(\beta) & =Z_{i}(\beta) L(\mathcal{O}) \\
\exp (\Lambda K) L(\mathcal{O}) \exp (-\Lambda K) & =\exp (\Lambda s(\mathcal{O})) L(\mathcal{O})
\end{aligned}
$$


where $s(\mathcal{O})$ denotes the Lorentz spin of the operator $\mathcal{O}$.

If one has these objects, then, as shown by Lukyanov [16], the functions

$$
F_{i_{1}, \ldots i_{n}}\left(\beta_{1}, \ldots, \beta_{n}\right)=\operatorname{Tr}_{\pi_{Z}}\left[\exp (2 \pi i K) L(\mathcal{O}) Z_{i_{n}}\left(\beta_{n}\right) \ldots Z_{i_{1}}\left(\beta_{1}\right)\right],
$$

are solutions of the form-factor axioms.

We now have to give the definition of the dual operators which give us the map $L$ representing the local operators of the model. We introduce the scalar field $\phi^{\prime}(\alpha)$ satisfying the same relations as $\phi$ together with its associated field $\bar{\phi}^{\prime}(\alpha)$. (We give a closer definition of these fields shortly when we introduce an ultraviolet regularization for the free field system.) With the help of these new fields we define the dual fields as follows:

$$
\begin{aligned}
& \Lambda_{-}(\alpha)=U^{\prime}(\alpha), \\
& \Lambda_{0}(\alpha)=-\frac{i}{\sqrt{2 \cos \left(\frac{\pi}{\xi+1}\right)}}\left[q^{\prime} \chi^{\prime} U^{\prime}(\alpha)-q^{\prime-1} U^{\prime}(\alpha) \chi^{\prime}\right], \\
& \Lambda_{+}(\alpha)=\frac{1}{2 \cos \left(\frac{\pi}{\xi+1}\right)}\left[q^{\prime} \chi^{\prime 2} U^{\prime}(\alpha)-\left(q^{\prime}+q^{\prime-1}\right) \chi^{\prime} U^{\prime}(\alpha) \chi^{\prime}+q^{\prime-1} U^{\prime}(\alpha) \chi^{\prime 2}\right] .
\end{aligned}
$$

The dual vertex operators $U^{\prime}$ and the $\bar{V}^{\prime}$ required for $\chi^{\prime}$, satisfy similar relations as the unprimed ones, but with the function $\bar{g}$ changed to

$$
\bar{g}^{\prime}(\alpha)=\kappa^{\prime 2} \frac{i \alpha}{\pi(\xi+1)} \frac{\Gamma\left(1-\frac{1}{(\xi+1)}+\frac{i \alpha}{\pi(\xi+1)}\right)}{\Gamma\left(\frac{1}{(\xi+1)}+\frac{i \alpha}{\pi(\xi+1)}\right)} .
$$

The parameter $q^{\prime}$ equals $\exp \left[-i \pi \frac{\xi}{\xi+1}\right]$.

One can also calculate all the normal ordering relations involving the primed and unprimed operators merely by using the relation

$$
\bar{V}^{\prime}(\delta) V(\beta)=\rho \bar{\rho}^{\prime} u(\beta-\delta): \bar{V}^{\prime}(\delta) V(\beta):
$$

where the function $u$ is given by

$$
u(\beta)=k \frac{i \beta}{2 \pi},
$$

with $k$ a constant.

Now one writes down the functions

$$
\mathcal{F}_{j_{1}, \ldots j_{l}}^{i_{1}, \ldots i_{k}}\left(\alpha_{1}, \ldots \alpha_{k} \mid \beta_{1}, \ldots \beta_{n}\right)=\operatorname{Tr}_{\pi_{Z}}\left[\exp (2 \pi i K) \Lambda_{i_{k}}\left(\alpha_{k}\right) \ldots \Lambda_{i_{1}}\left(\alpha_{1}\right) Z_{j_{l}}\left(\beta_{l}\right) \ldots Z_{j_{1}}\left(\beta_{1}\right)\right],
$$

then these functions have to be expanded as

$$
\mathcal{F}_{j_{1}, \ldots j_{l}}^{i_{1}, \ldots i_{k}}\left(\alpha_{1}, \ldots \alpha_{k} \mid \beta_{1}, \ldots \beta_{n}\right)=\sum_{\left\{s_{j}\right\}} F^{\prime i_{1}, \ldots i_{k}}\left(\alpha_{1}, \ldots \alpha_{k} \mid\left\{s_{j}\right\}\right) F_{j_{1}, \ldots j_{l}}\left(\left\{s_{j}\right\} \mid \beta_{1}, \ldots \beta_{n}\right) \times \exp \left(s_{1} \alpha_{1}\right) \ldots \exp \left(s_{k} \alpha_{k}\right),
$$

where the functions $F$ are the required solutions of the form-factor axioms. This means that the functions $\mathcal{F}$ play the role of generating functions for the form-factors of local operators. They describe $n$-particle form-factors of infinitely many operators which may be considered as descendents of the first one in the sense of [9].

The actual calculation of the matrix elements and the traces proceeds through the procedure described in [16]. The free field construction needs a regularization; we choose an ultraviolet cut-off by taking the rapidity interval finite

$$
-\frac{\pi}{\epsilon}<\beta<\frac{\pi}{\epsilon}
$$

Then we introduce the mode expansion of the free field $\phi$ as:

$$
\phi_{\epsilon}(\beta)=\sqrt{s}(Q-i \beta P)+\sum_{k \neq 0} \frac{a_{k}}{i \sinh (\pi k \epsilon)} \exp (i k \epsilon \beta),
$$

with 


$$
s=\frac{\xi+1}{2 \xi} .
$$

The oscillator modes satisfy the commutation relation

$$
\left[a_{k}, a_{l}\right]=\frac{\sinh \frac{\pi k \epsilon}{2} \sinh \pi k \epsilon}{k} \frac{\sinh \frac{\pi k \epsilon}{2}(\xi+1)}{\sinh \frac{\pi k \epsilon}{2} \xi} \delta_{k,-l},
$$

while for the zero modes we have the usual canonical ones:

$$
[P, Q]=-i .
$$

The two-point function and the commutation relations for this field can be calculated and it can be seen that in the limit $i \rightarrow 0$ we recover the relations satisfyed by the field $\phi$ (see eq. (3.7)). The dual field is given by

$$
\phi_{\epsilon}^{\prime}(\alpha)=-\sqrt{s^{\prime}}(Q-i \alpha P)+\sum_{k \neq 0} \frac{a_{k}^{\prime}}{i \sinh (\pi k \epsilon)} \exp (i k \epsilon \alpha), s^{\prime}=\frac{\xi}{2(\xi+1)},
$$

with the oscillators $a_{k}^{\prime}$ defined by the relation

$$
a_{k}^{\prime} \sinh \frac{\pi k \epsilon}{2}(\xi+1)=a_{k} \sinh \frac{\pi k \epsilon}{2} \xi .
$$

We introduce the conventional notation used in conformal field theory

$$
\alpha_{+}=-1 / \alpha_{-}=\sqrt{\frac{\xi+1}{\xi}} .
$$

The dual set of operators can actually be obtained by the substitution $\xi \rightarrow-1-\xi$, which is known to have an analogy in conformal field theory (see Lukyanov [16] for details). The space where the operators and their duals act is defined to be

$$
\pi_{Z}^{\epsilon}=\bigoplus_{l, l^{\prime} \in Z} F_{\frac{\alpha_{+} l+\alpha_{-} l^{\prime}}{\sqrt{2}}}
$$

where $F_{p}$ denotes the Fock space built up with the help of the creation operators (as usual, the oscillator modes with negativ indices) from the ground state $|p\rangle$ which satisfies:

$$
P|p\rangle=p|p\rangle .
$$

The operator $K$, of the Lorentz boost is given by

$$
K_{\epsilon}=i \epsilon H+\frac{\alpha_{+}+\alpha_{-}}{\sqrt{2}} P,
$$

where

$$
H=\frac{P^{2}-p_{0}^{2}}{2}+\sum_{k=1}^{\infty} \frac{k^{2}}{\sinh \frac{\pi k \epsilon}{2} \sinh \pi k \epsilon} a_{-k}^{\prime} a_{k} .
$$

Formally we can write $\pi_{Z}=\lim _{\epsilon \rightarrow 0} \pi_{Z}^{\epsilon}$. This should be understood in the following sense: we make all calculations with the regularized operators and in the end take the limit $\epsilon \rightarrow 0$.

\section{SAUSAGE MODEL FROM BOOTSTRAP FUSION OF SINE-GORDON MODEL}

In this section we describe how to obtain the sausage model S-matrix introduced in section II from that of the sine-Gordon theory. First we introduce some graphical notations. We denote the S-matrix by a cross: 


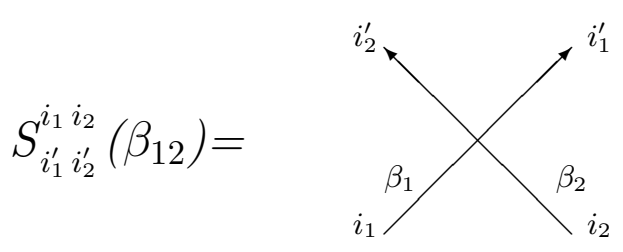

This should be understood as follows: the lines are vector spaces carrying the internal indices of the particles. The S-matrix acts in the tensor product of two such spaces and carries an argument which is the rapidity difference of the two scattering particle. The rapidities are associated to the lines of the diagram. Each segment carries an index and repeated indices must be summed over. The order of the matrix products is represented by the arrows. As an example, the Yang-Baxter equation in these notations can be written as (omitting the rapidities of the lines, which causes no confusion)

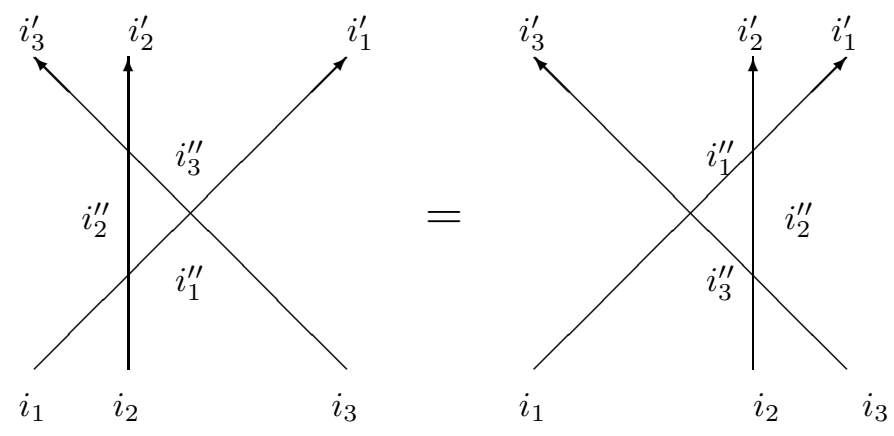

which means

$$
S_{i_{2}^{\prime} i_{3}^{\prime}}^{i_{2}^{\prime \prime} i_{3}^{\prime \prime}}\left(\beta_{23}\right) S_{i_{1}^{\prime} i_{3}^{\prime \prime}}^{i_{1 \prime}^{\prime \prime} i_{3}}\left(\beta_{13}\right) S_{i_{1}^{\prime \prime} i_{2}^{\prime \prime}}^{i_{1} i_{2}}\left(\beta_{12}\right)=S_{i_{1}^{\prime} i_{2}^{\prime}}^{i_{1}^{\prime \prime} i_{2}^{\prime \prime}}\left(\beta_{12}\right) S_{i_{1}^{\prime \prime} i_{3}^{\prime}}^{i_{1} i_{3}^{\prime \prime}}\left(\beta_{13}\right) S_{i_{2}^{\prime \prime} i_{3}^{\prime \prime}}^{i_{2} i_{3}}\left(\beta_{23}\right)
$$

The bootstrap fusion means the following formal operation on the level of the ZF operators

$$
\mathcal{Z}_{I}(\beta)=C_{I}^{i_{1} i_{2}} Z_{i_{1}}\left(\beta_{1}+\frac{i \pi}{2}\right) Z_{i_{2}}\left(\beta_{2}-\frac{i \pi}{2}\right)
$$

where $C_{I}^{i_{1} i_{2}}$ denotes the appropriate Clebsh-Gordan coefficients for the internal indices. In our case they are just the coefficients for the spin-1 representation in the product of two spin-1/2 representations. The nonzero elements are:

$$
C_{+}^{++}=C_{-}^{--}=1, C_{0}^{+-}=\frac{1}{\sqrt{q+q^{-1}}} q^{-1 / 2}, C_{0}^{-+}=\frac{1}{\sqrt{q+q^{-1}}} q^{1 / 2}
$$

Eqn. (4.2) is formal since the rapidity difference just corresponds to the position of the annihilation poles and so there are potential divergences. Fortunately, in the case we investigate these singularities drop out and we will be able to define the ZF operators corresponding to the RHS of the equation 4.2 in the free field representation. We will denote this operation graphically by

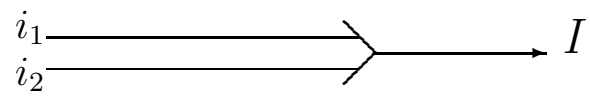

Then we can write down the graphical representation for the S-matrix obtained from the bootstrap fusion:

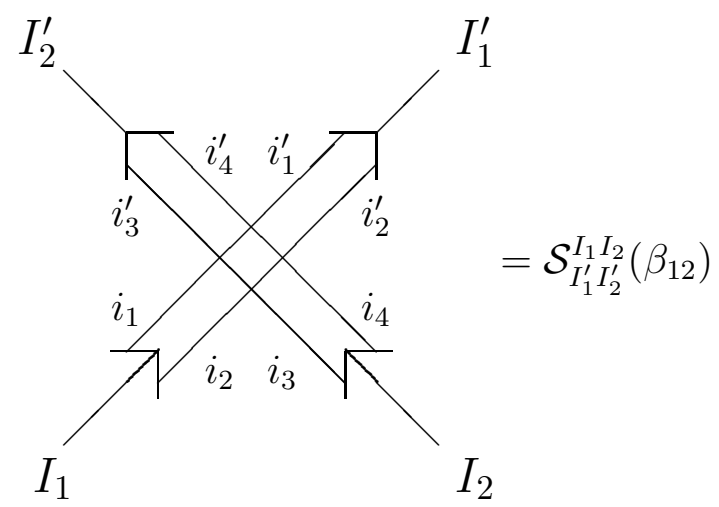


This diagram should be read as follows: two incoming particle in the triplet representation is converted (using the fusion formula 4.2) into two pairs of doublets and then scattered on each other (this means four crossings ie. four S-matrix factors). After that, the triplet part is projected out using again the fusion formula. However, care must be taken, since in the case of the incoming particles we make an embedding of the triplet representation into the product of the two doublet representations, while in the case of the outgoing particles we go the other way via projection. These two operations are adjoint to each other and differ not only by transposition of matrices, but (and here comes an essential difference from the $\mathrm{O}(3)$ sigma model case treated in [1]) also differ by a substitution $q \rightarrow 1 / q$, which is here just a complex conjugation since $|q|=1$. We can write the fusion equation in the following form (using the redefinitions 2.9, 2.10 and 2.15)

$$
\hat{\mathcal{S}}_{I_{1}^{\prime} I_{2}^{\prime}}^{I_{1} I_{2}}\left(\beta_{12}\right)=C_{I_{1}^{\prime}}^{i_{1}^{\prime} i_{2}^{\prime}} C_{I_{2}^{\prime}}^{i_{3}^{\prime} i_{4}^{\prime}} \hat{S}_{i_{1}^{\prime} i_{4}^{\prime}}^{i_{1}^{\prime \prime} i_{4}^{\prime \prime}}\left(\beta_{12}+i \pi\right) \hat{S}_{i_{1} i_{3}^{\prime \prime}}^{i_{1}^{\prime \prime} i_{3}^{\prime}}\left(\beta_{12}\right) \hat{S}_{i_{2}^{\prime} i_{4}^{\prime \prime}}^{i_{2}^{\prime \prime} i_{4}}\left(\beta_{12}\right) \hat{S}_{i_{2}^{\prime \prime} i_{3}^{\prime \prime}}^{i_{2} i_{3}}\left(\beta_{12}-i \pi\right) \tilde{C}_{i_{1} i_{2}}^{I_{1}} \tilde{C}_{i_{3} i_{4}}^{I_{2}}
$$

The coefficients $C$ and $\tilde{C}$ are given in (4.3). We remark that it is necessary to use the redefined S-matrices because to apply the quantum group Clebsh-Gordan coefficients we have to go to the one-particle basis which is at the same time the properly normalized basis of the quantum group representation.

The proof of the fusion relation between the sine-Gordon and sausage model S-matrices proceeds as follows. The sine-Gordon S-matrix acts in the tensor product of two spaces; we can expand it in terms of tensor products of Pauli matrices acting in one space as follows:

$$
\begin{aligned}
S_{0}(\beta) \frac{1}{2 \sinh \left(\frac{\beta-i \pi}{\xi}\right)}[ & I_{1} \otimes I_{2}\left(\sinh \left(\frac{\beta-i \pi}{\xi}\right)-\sinh \left(\frac{\beta}{\xi}\right)\right)+\sigma_{1}^{3} \otimes \sigma_{2}^{3}\left(\sinh \left(\frac{\beta-i \pi}{\xi}\right)+\sinh \left(\frac{\beta}{\xi}\right)\right) \\
& \left.-\sinh \left(\frac{i \pi}{\xi}\right)\left(\sigma_{1}^{1} \otimes \sigma_{2}^{1}+\sigma_{1}^{2} \otimes \sigma_{2}^{2}\right)\right] .
\end{aligned}
$$

The redefinition means changing the Pauli matrices by rapidity-dependent factors. The product of the four S-matrices can be written in the tensor product of four two-dimensional spaces, each S-matrix acting only in two of them: eg. the first S-matrix in the spaces 1 and 4, the second one in the spaces 1 and 3 and so on. Then one can embed the Clebsh-Gordan coefficients into this 16 dimensional space and carry out the matrix multiplication. The special structure reflected in the graphical presentation of the formula greatly simplifies the required computation. The relation between the parameters of the two models turns out to be $\lambda=1 / \xi$.

Now we turn to the fusion of the ZF operators. We want to give a meaning to the formula 4.2. We interpret it as the limit

$$
\hat{\mathcal{Z}}_{I}(\beta)=\lim _{\delta \rightarrow 0} C_{I}^{i_{1} i_{2}} \hat{Z}_{i_{1}}\left(\beta_{1}+\frac{i \pi}{2}+\delta\right) \hat{Z}_{i_{2}}\left(\beta_{2}-\frac{i \pi}{2}-\delta\right) .
$$

Remarkably, all the singularities cancel and the result is a very simple formula for in terms of the free field representation:

$$
\begin{aligned}
& \mathcal{Z}_{+}(\beta)=\exp \left(-\frac{\beta}{\xi}\right) U(\beta), \\
& \mathcal{Z}_{0}(\beta)=\frac{i}{\sqrt{2 \cos \left(\frac{\pi}{\xi}\right)}}\left[q \chi U(\beta)-q^{-1} U(\beta) \chi\right], \\
& \mathcal{Z}_{-}(\beta)=-\frac{1}{\cos \left(\frac{\pi}{\xi}\right)} \exp \left(\frac{\beta}{\xi}\right)\left[q \chi^{2} U(\beta)-\left(q+q^{-1}\right) \chi U(\beta) \chi+q^{-1} U(\beta) \chi^{2}\right] .
\end{aligned}
$$

The proof of this result can be found in Appendix A, where we also outline the direct calculation of the scattering relations. They prove to be identical to the sausage model ZF algebra:

$$
\mathcal{Z}_{I}\left(\beta_{1}\right) \mathcal{Z}_{J}\left(\beta_{2}\right)=\mathcal{S}_{I J}^{K L}\left(\beta_{12}\right) \mathcal{Z}_{L}\left(\beta_{2}\right) \mathcal{Z}_{K}\left(\beta_{1}\right) .
$$

However, just as in the case of the $\mathrm{O}(3)$ sigma model [i], the operator product does not have the right kinematical poles. Instead of having them at rapidity difference $i \pi$, it turns out that the singularities are situated at $2 i \pi$ (see Appendix (Q). Nevertheless, if we write down the trace formula 3.25, it will solve the cyclicity (by the definition of the trace) and Watson's axiom. The analytic structure will be corrected by a procedure similar to that in [1]. 


\section{EVALUATION OF THE EXPRESSIONS FOR THE FORM-FACTORS}

We have to calculate the traces required for obtainig the integrand of the representation for the form-factors. Using the coherent state representation for the traces, we find the result:

$$
\begin{aligned}
& \operatorname{Tr}_{\pi_{Z}}\left[\exp (2 \pi i K) U^{\prime}\left(\alpha_{k}\right) \ldots U^{\prime}\left(\alpha_{1}\right) \bar{V}^{\prime}\left(\delta_{p}\right) \ldots \bar{V}^{\prime}\left(\delta_{1}\right) U\left(\beta_{n}\right) \ldots U\left(\beta_{1}\right) \bar{V}\left(\gamma_{r}\right) \ldots \bar{V}\left(\gamma_{1}\right)\right]= \\
& \mathcal{C}_{1}^{-n} \mathcal{C}_{2}^{\frac{n+r}{2}} \mathcal{C}_{2}^{\prime \frac{p+k}{2}} 2^{-r-n} i^{n+p+k} \eta^{r} \eta^{\prime-p-k} \delta_{n r} \delta_{p k} \exp \left(\frac{1}{\xi}\left[\sum_{j=1}^{n} \beta_{j}-\sum_{j=1}^{r} \gamma_{j}\right]+\frac{1}{\xi+1}\left[\sum_{j=1}^{p} \delta_{j}-\sum_{j=1}^{k} \alpha_{j}\right]\right) \times \\
& \prod_{i<j} \bar{G}\left(\beta_{i}-\beta_{j}\right) \prod_{i<j} \bar{G}\left(\gamma_{i}-\gamma_{j}\right) \prod_{i, j} \bar{G}^{-1}\left(\gamma_{i}-\beta_{j}\right) \prod_{i<j} \bar{G}^{\prime}\left(\alpha_{i}-\alpha_{j}\right) \prod_{i<j} \bar{G}^{\prime}\left(\delta_{i}-\delta_{j}\right) \prod_{i, j} \bar{G}^{\prime-1}\left(\delta_{i}-\alpha_{j}\right) \times \\
& \prod_{i, j} \bar{H}\left(\beta_{i}-\alpha_{j}\right) \prod_{i, j} \bar{H}^{-1}\left(\beta_{i}-\delta_{j}\right) \prod_{i, j} \bar{H}\left(\gamma_{i}-\delta_{j}\right) \prod_{i, j} \bar{H}^{-1}\left(\gamma_{i}-\alpha_{j}\right) .
\end{aligned}
$$

Here we defined the new functions as:

$$
\begin{aligned}
& \bar{G}(\beta)=-\frac{\mathcal{C}_{2}}{4} \xi \sinh \left(\frac{\beta+i \pi}{\xi}\right) \sinh \beta, \\
& \bar{G}^{\prime}(\alpha)=-\mathcal{C}_{2}^{\prime} \frac{\sinh \alpha}{(\xi+1) \sinh \left(\frac{\alpha+i \pi}{\xi+1}\right)}, \\
& \bar{H}(\alpha)=-\frac{2}{\cosh \alpha}
\end{aligned}
$$

and the constants are given by:

$$
\begin{aligned}
& \mathcal{C}_{1}=\exp \left[-\int_{0}^{\infty} \frac{d t}{t} \frac{\sinh ^{2} \frac{t}{2} \exp (-t)}{\sinh 2 t \cosh t} \frac{\sinh t(\xi-1)}{\sinh t \xi}\right], \\
& \mathcal{C}_{2}=\exp \left[4 \int_{0}^{\infty} \frac{d t}{t} \frac{\sinh ^{2} \frac{t}{2} \exp (-t)}{\sinh 2 t \cosh t} \frac{\sinh t(\xi-1)}{\sinh t \xi}\right], \\
& \mathcal{C}_{2}^{\prime}=\exp \left[-4 \int_{0}^{\infty} \frac{d t}{t} \frac{\sinh ^{2} \frac{t}{2} \exp (-t)}{\sinh 2 t \cosh t} \frac{\sinh t \xi}{\sinh t(\xi+1)}\right] .
\end{aligned}
$$

The trace over the zero modes contains an infinite constant which we have set equal to unity.

We also have to specify the integration over the variables of the screening charges. We face the following problem: when we take the integration contour as given in the sine-Gordon model (see in [16]) and try to take the limit which gives the sausage model, new double poles arise in the integration over the $\gamma_{i}$ variables whose positions depend on the $\beta_{j}$ variables. This phenomenon was already noticed by Smirnov [7] for the case of the $\mathrm{O}(3)$ sigma model. The contour gets pinched by the two poles approaching each other and the integrals diverge. This means that the limit to the sausage model is singular. To deal with the integral we introduce the prescription that one should take the coefficient of the most divergent term in the integral.

We are now to give a justification of the prescription and explain the appearance of double poles in the "finite temperature" expressions, while they are not present in the vacuum expectation values, ie. the "zero-temperature" matrix elements.

The basic picture is as follows. Taking the trace means imposing the periodicity equation (or KMS condition) included in the form-factor axioms. What happens is that while in calculating matrix elements we use Green functions of the scalar field on the plane, here we impose the periodcity condition. The original function $\bar{g}(\beta)$ has poles at the rapidity difference 0 and $i \pi$, which will have all their images under the KMS and the Watson's symmetry condition. They can be viewed as mirror charges introduced to satisfy the boundary conditions for $\bar{G}(\beta)$, which is the analogue of finite-temperature Green function, if we take $\bar{g}$ to be the analogue of zero temperature one. Let us for simplicity take the case of the $\mathrm{O}(3)$ model, which means $\xi=\infty$. We deform the trace by substituting $\exp (2 \pi i K)$ by $\exp ((2 \pi+\zeta) i K)$, where $\zeta$ is a small positive number. This means a deformation of form-factors which is the same as considered in [19]. The deformed form-factor wiil obey Watson's symmetry property and a deformed KMS condition: 


$$
F_{i_{1}, \ldots, i_{n}}\left(\beta_{1}, \ldots, \beta_{n}+(2 \pi+\zeta) i\right)=F_{i_{n}, i_{1}, \ldots, i_{n-1}}\left(\beta_{n}, \beta_{1}, \ldots, \beta_{n-1}\right) .
$$

Looking at the explicit form of the function $\bar{G}$, when $\zeta>0$, which is (up to a normalization factor)

$$
\bar{G}^{(\zeta)}(\beta)=\frac{(\beta+i \pi) \sinh \left(\frac{\pi \beta}{2 \pi+\zeta}\right)}{\Gamma\left(1-\frac{\pi-i \beta}{2 \pi+\zeta}\right) \Gamma\left(1-\frac{\pi+i \beta}{2 \pi+\zeta}\right)},
$$

(for the calculation see Appendix $\mathrm{B}$ ), we see that the double pole in $\bar{G}^{-1}$ (which is relevant for the integrand of the contour integral over $\gamma_{i}$ ) arises when $\zeta \rightarrow 0$. However, the operator product singularities are the same for finite $\zeta$, ie we have a pole at $\beta_{12}=2 \pi i$ and all its periodic images, and the generating function is regular at $\beta_{12}=\pi i$. So we take the leading singular term of it when $\zeta \rightarrow 0$. It will automatically obey the (undeformed) KMS condition and Watson's property. But its behaviour at the positions of rapidity difference $i \pi$ changes. In Appendix B it is shown that the nonsingular behaviour of the operator product at these points is to be attributed to a zero in the Green function cancelling the pole coming from the pinch of the contour integral. But the same effect, which doubles the pole in the integrand, also doubles this zero in the factors $\bar{G}\left(\beta_{i}-\beta_{j}\right)$ and so the net result is a function having simple poles at $\beta_{i j}=0,2 \pi i$ and simple zeros at $\beta_{i j}=\pi i$. This is displayed well in the structure of the generating function for the $\mathrm{O}(3)$ current, calculated in [1], which we remind here:

$$
\frac{2 i \pi\left(\beta_{21}+i \pi\right)}{\left(\beta_{21}+2 i \pi\right) \beta_{21}}\left[\frac{1}{\cosh \left(\beta_{1}-\alpha\right)}+\frac{1}{\cosh \left(\beta_{2}-\alpha\right)}\right] \tanh ^{2} \frac{\beta_{21}}{2} .
$$

To deal with this problem, we saw in [1], that we had to introduce a $2 \pi i$ periodic, symmetric function, which killed the poles at 0 and $2 \pi i$ and turned the zero at $\pi i$ into a pole. This was the function (here written for n-particle case)

$$
\prod_{1 \leq i<j \leq n} \tanh ^{2} \frac{\beta_{i j}}{2}
$$

The end result for the generating function of the two-particle form-factors of the current (and its descendents) is therefore

$$
\frac{i \pi m}{8} \epsilon^{A B C} \frac{\beta_{12}-i \pi}{\beta_{12}\left(2 i \pi-\beta_{12}\right)} \tanh ^{2} \frac{\beta_{12}}{2}\left(\frac{1}{\cosh \left(\alpha-\beta_{1}\right)}+\frac{1}{\cosh \left(\alpha-\beta_{2}\right)}\right) .
$$

taking into account the proper normalization factors to get the correct value of the charges on particle states. In the light of the argument given above, we expect this to be a general prescription to get the right structure of kinematical poles.

Although in the above discussion we took $\xi=\infty$, it carries over to finite values of $\xi$. In this case additional poles are introduced (both in $\bar{g}$ and $\bar{G}$ ) but they are outside the strip since we choose $\xi>2$ in order to be in the regime where the sausage model has no bound state poles. Taking suitably small $\zeta$ it is possible to draw the contours in a way which avoids these additional singularities.

For the contours of integration over the variables $\delta_{j}$, no similar problem arises and we have to draw the contours just as for the vacuum matrix elements but they must lie within the strip $-i \pi<\delta_{j}<i \pi$. This completes our prescription of how to write down generating functions for the form-factors of the sausage model.

We would like to note that in the case of finite $\xi$, the correct analogue of the above generating function is not just a simple continuation to finite $\xi$ [1]. The expression

$$
\operatorname{Tr}_{\pi_{Z}}\left[\Lambda_{-}(\alpha) \mathcal{Z}_{0}\left(\beta_{2}\right) \mathcal{Z}_{+}\left(\beta_{1}\right)\right]
$$

turns out to be zero due to the Kronecker delta arising from the zero mode trace. The correct analogue would be to take eg.

$$
\operatorname{Tr}_{\pi_{Z}}\left[\Lambda_{0}(\alpha) \mathcal{Z}_{+}\left(\beta_{2}\right) \mathcal{Z}_{-}\left(\beta_{1}\right)\right]
$$

If one writes down this expression, one gets a complicated integral formula which however can be seen to yield the correct $\mathrm{O}(3)$ value in the limit $\xi \rightarrow 0$ (as well as all other formulas for form-factors written down using the general expression (5.1)).

The case $\xi=\infty$ is special since in that case the appropriate factor is $\delta_{2 n-2 r+2 p-2 k}$, which allows much more nonzero solutions [1]. The reason for that is that the zero-mode trace enforces momentum conservation for the free 
field. While in the $\mathrm{O}(3)$ case $\alpha_{+}=-\alpha_{-}$and the momentum cancellation is possible between the vertex operator and their duals, for generic $\xi$ this is not the case: they have to cancel separately. Since we have actually Two quantum groups, one with $q=\exp i \pi \alpha_{+}^{2}$ for the vertex operators $\mathcal{Z}$ and one with $q^{\prime}=\exp i \pi \alpha_{-}^{2}$ for their duals, the condition which is imposed is actually that we should have operators under the trace which are singlets separately under each of them. This is very much in the spirit of Smirnov's work [9] and resembles his notion of the "main set of solutions" (which are the one which have their counterparts when deforming away from $q=-1$ ). If $\xi$ is rational, however, some cancellations happen and the space of the solutions increases, suggesting an analogy with the reduction of sine-Gordon theory at rational values of the coupling, corresponding to some well-known changes in the representation theory of the quantum group when the value of $q$ equals a root of unity [20].

\section{CONCLUSIONS AND FURTHER QUESTIONS}

In this paper we were able to show that there exists a free field representation for the sausage model, very similar to the one found in [1] for the $\mathrm{O}(3)$ case. We could also identify the origin of the problem with the kinematical singularities and the singularity in the fusion limit: the first is related to the fact that the operator product singularities of the fused ZF operators are not the correct ones, while the second is due to the to the additional poles introduced when taking the "finite-temperature" Green function instead of the vacuum one. Both of them is ultimately related to the zero of the function $\bar{g}=\beta_{21}\left(\beta_{21}+i \pi\right)$ at $\beta_{12}=i \pi$. We would like to remark that the $\tanh ^{2}$ factors which were put in by hand can be considered as arising from vertex operators of usual conformal free scalar fields with the two-point function $-\log \beta_{12}$. Since such vertex operators will have trivial braiding, one can write down a tensor product representation by tensoring our vertex operators with the conformal free field vertex operators. Then the expressions for the form-factors will automatically include the correction factors for the kinematical poles. However, all our attempts to find an "irreducible" (not tensor product) representation have failed so far. For this one has to change the $\bar{g}$ function, while retaining its property

$$
\frac{\bar{g}(-\beta)}{\bar{g}(\beta)}=\frac{\sinh \left(\frac{\beta-i \pi}{\xi}\right)}{\sinh \left(\frac{\beta+i \pi}{\xi}\right)},
$$

in such a way that we get the right operator product structure. One has to notice that the above condition determines $\bar{g}$ only up to multiplication by an even function. However it does not seem possible to find such a function, although we have no proof that such a function cannot exist. We simply have been unable to construct it so far. The solution, which takes tensor product representation does not seem to be superior to the procedure of simply introducing the additional factors.

We have obtained a procedure with which one can (in principle) calculate form-factors of the sausage model. The main question, however, is to find a general procedure with which one can associate a similar free field representation to any factorizable S-matrix and in this way find the solutions for the form-factor axioms. The other question is whether the solutions obtained from the free field representation form a complete set. If this is true, then the method may provide a major step towards understanding the operator content of the models. We also want to mention in this context the relations to the work of Smirnov [9]. It would be interesting to analyse the form-factors from the point of KZ equations and following the lines of 10 12 to obtain a counting of the local fields in the model. It also seems worthwile to examine the rational values of $\xi$ and to see what sort of structure emerges in that case.

\section{ACKNOWLEDGMENTS}

The authors would like to thank M. R. Niedermaier for useful discussion. This work was partially supported by the Hungarian National Science and Research Foundation.

\section{APPENDIX A: PROOF OF THE BOOTSTRAP FUSION AND THE ZAMOLODCHIKOV-FADDEEV ALGEBRA RELATIONS}

In this appendix we outline the proof of the bootstrap fusion and the ZF relation. Starting with the bootstrap fusion, the only nontrivial relation to prove is: 


$$
\hat{\mathcal{Z}}_{+}(\beta)=C_{--}^{-} \hat{Z}+_{i_{1}}\left(\beta_{1}+\frac{i \pi}{2}\right) \hat{Z}+_{i_{2}}\left(\beta_{2}-\frac{i \pi}{2}\right) .
$$

Writing out in details:

$$
\begin{aligned}
& \exp \left(\frac{\beta}{\xi}\right)\left[q \chi^{2} U(\beta)-\left(q+q^{-1}\right) \chi U(\beta) \chi+q^{-1} U(\beta) \chi^{2}\right]= \\
& \exp \left(\frac{\beta_{+}}{2 \xi}\right)\left(q^{\frac{1}{2}} \chi V\left(\beta_{+}\right)-q^{-\frac{1}{2}} V\left(\beta_{+}\right) \chi\right) \exp \left(\frac{\beta_{-}}{2 \xi}\right)\left(q^{\frac{1}{2}} \chi V\left(\beta_{-}\right)-q^{-\frac{1}{2}} V\left(\beta_{-}\right) \chi\right),
\end{aligned}
$$

where $\beta_{ \pm}=\beta \pm i \pi / 2$.

First, we prove the equality for the integrands using the normal ordering procedure. We symmetrize in the arguments of the screening charges, since they are to be integrated out. We get the relation:

$$
\begin{aligned}
& \left(q \bar{g}^{-1}\left(\beta-\gamma_{1}\right) \bar{g}^{-1}\left(\beta-\gamma_{2}\right)-\left(q+q^{-1}\right) \bar{g}^{-1}\left(\gamma_{1}-\beta\right) \bar{g}^{-1}\left(\beta-\gamma_{2}\right)+q^{-1} \bar{g}^{-1}\left(\gamma_{1}-\beta\right) \bar{g}^{-1}\left(\gamma_{2}-\beta\right)\right) \bar{g}\left(\gamma_{2}-\gamma_{1}\right)+ \\
& \left(\text { similar term with } \gamma_{1} \leftrightarrow \gamma_{2}\right)= \\
& \bar{g}\left(\gamma_{2}-\gamma_{1}\right) w\left(\gamma_{2}-\beta_{+}\right) w\left(\beta_{-}-\gamma_{1}\right)\left(q^{\frac{1}{2}} w\left(\beta_{+}-\gamma_{1}\right)-q^{-\frac{1}{2}} w\left(\gamma_{1}-\beta_{+}\right)\right)\left(q^{\frac{1}{2}} w\left(\beta_{-}-\gamma_{2}\right)-q^{-\frac{1}{2}} w\left(\gamma_{2}-\beta_{-}\right)\right)+ \\
& \left(\text {similar term with } \gamma_{1} \leftrightarrow \gamma_{2}\right),
\end{aligned}
$$

which can be proven after some elementary, but lengthy calculations. Then one proves the equality the integrals, which amounts to checking that the contours can be deformed into each other.

For the proof of the ZF relations one actually uses only the ratio $\bar{g}(\beta) / \bar{g}(-\beta)$. Hence one can substitute the functions $\bar{g}$ by other functions having the same ratio, eg. $\bar{G}$ in the proof. The proof is essentially the same as presented in [1]. In that work, we could only work out the proof up to the identities containing two screening charges. Since then, improving our MAPLE algorithms, we have been able to prove the ZF relations for all combinations of the $\mathcal{Z}$ operators, including those with three and four screening charges, in the general sausage model case. The procedure is very similar to the proof of the fusion relation, but the formulas are even more lengthy and we do not attempt to describe it here. For the $\mathrm{O}(3)$ case it was discussed in some details in [1].

\section{APPENDIX B: EVALUATION OF TRACES USING COHERENT OSCILLATOR METHODS}

Here we show how to calculate the traces involved in the form-factors using coherent oscillator methods. We will take a very simple example and calculate in the $\mathrm{O}(3)$ limit, when $\xi=\infty$. We will also calculate only the non-zero mode part of the trace, since it is the most interesting contribution when we discuss the analytical structure in the main text. We will take the case when the regularizing parameter $\zeta$ is finite and reproduce the function $\left.\bar{G}^{(} \zeta\right)$ used in the main text. The expression we want to calculate is:

$$
\operatorname{Tr}\left(e^{\omega i K} e^{i \bar{\phi}\left(\beta_{1}\right)} e^{i \bar{\phi}\left(\beta_{2}\right)}\right)
$$

with $\omega=2 \pi-\zeta$ introduced for convenience. We omitted the indices $\epsilon$ from $K$ and $\bar{\phi}$ since it is clear that we use their regularized version and this will cause no confusion.

We use the following redefinitions. First, we change our oscillator to have the usual normalization:

$$
\left[\alpha_{n}, \alpha_{m}\right]=m \delta_{m+n .0} .
$$

These are related to $a_{m}$ in the following way:

$$
a_{m}=\sqrt{N_{m}(\epsilon)} \alpha_{m}, \quad a_{-m}=\sqrt{N_{m}(\epsilon)} \alpha_{-m},
$$

where

$$
N_{m}(\epsilon)=\frac{\sinh \frac{\pi m \epsilon}{2} \sinh \pi m \epsilon}{m} e^{\frac{\pi m \epsilon}{2}} .
$$

As usual, the operators $\alpha_{m}$ and $\alpha_{-m}$ are Hermitian conjugates. The non-zero mode part of the operator $K$ looks very familiar:

$$
K=i \epsilon \sum_{m=1}^{\infty} m \alpha_{-m} \alpha_{m}+\text { zero mode terms }
$$


We normal order under the trace to get:

$$
\left.\bar{g}_{(} \beta_{21}\right) \operatorname{Tr}\left(e^{\omega i K}: e^{i \bar{\phi}\left(\beta_{1}\right)} e^{i \bar{\phi}\left(\beta_{2}\right)}:\right),
$$

and put in the explicit expressions (3.9), (3.28). Observe that the trace factorizes into independent pieces over each harmonic oscillator. We evaluate it in the basis of coherent states. The relevant formulas are:

$$
\begin{aligned}
\alpha \mid \lambda)=\lambda \mid \lambda), \quad\left(\lambda \mid \alpha^{\dagger}\right. & \left.=\left(\lambda\left|\bar{\lambda}, x^{\alpha^{\dagger} \alpha}\right| \lambda\right)=\mid x \lambda\right), \\
\operatorname{Tr} \mathcal{O} & =\int \frac{d \lambda d \bar{\lambda}}{\pi} e^{-\lambda \bar{\lambda}}(\lambda|\mathcal{O}| \lambda) .
\end{aligned}
$$

Here we omitted the mode index $m$ since the above formulas are the same for each oscillator mode. Performing the calculation for each mode separately, we get a Gaussian integral, which can be evaluated easily. Putting together all the contributions we find

$$
\bar{g}\left(\beta_{21}\right) \prod_{m=1}^{\infty}\left(1-e^{-\omega \epsilon m}\right)^{-1} \exp \left(-\frac{2 N_{m}(\epsilon) e^{-\omega \epsilon m}}{\sinh ^{2} \frac{\pi m \epsilon}{2}\left(1-e^{-\omega \epsilon m}\right)}\right) \prod_{m=1}^{\infty} \exp \left(-\frac{2 N_{m}(\epsilon) e^{-\omega \epsilon m} \cosh \left(i m \epsilon \beta_{12}\right)}{\sinh ^{2} \frac{\pi m \epsilon}{2}\left(1-e^{-\omega \epsilon m}\right)}\right)
$$

Now we substitute $N_{m}(\epsilon)$ and then expand

$$
\left(1-e^{-\omega \epsilon m}\right)^{-1}=\sum_{n=0}^{\infty}\left(e^{-\omega \epsilon n}\right)^{m}
$$

in the exponent. We can then sum up in $m$ using the formula

$$
\sum_{m=1}^{\infty} \frac{x^{m}}{m}=\log (1-x)
$$

Ignoring the rapidity independent parameters (which can be assembled into the coefficient $\mathcal{C}_{2}$ ), we arrive at an infinite product formula:

$$
\prod_{n=0}^{\infty}\left[\left(1-e^{-\alpha \epsilon n+(\pi-\alpha) \epsilon+i \epsilon m \beta_{12}}\right)\left(1-e^{-\alpha \epsilon n+(\pi-\alpha) \epsilon-i \epsilon m \beta_{12}}\right)\left(1-e^{-\alpha \epsilon n-\alpha \epsilon+i \epsilon m \beta_{12}}\right)\left(1-e^{-\alpha \epsilon n-\alpha \epsilon-i \epsilon m \beta_{12}}\right)\right] .
$$

Using the definition of the deformed gamma function

$$
\Gamma_{\epsilon}(x)=\left[1-e^{-2 \pi \epsilon}\right]^{1-x} \prod_{n=0}^{\infty} \frac{1-\exp (-2 \pi \epsilon(n+1))}{1-\exp (-2 \pi \epsilon(x+n))},
$$

and the fact, that for $\epsilon \rightarrow 0$ it tends to the usual gamma function, we get for $\bar{G}^{(\zeta)}\left(\beta_{12}\right)$ the expression (apart from constant factors):

$$
\frac{\left(\beta_{21}+i \pi\right) \sinh \left(\frac{\pi \beta_{21}}{\omega}\right)}{\Gamma\left(1-\frac{\pi}{\omega}+\frac{i \beta_{21}}{\omega}\right) \Gamma\left(1-\frac{\pi}{\omega}+\frac{i \beta_{21}}{\omega}\right)}
$$

which in the limit $\zeta \rightarrow 0$ gives back the $\xi=\infty$ case of 5.2 .

\section{APPENDIX C: A SAMPLE CALCULATION OF THE OPERATOR PRODUCT SINGULARITIES}

Here we sketch the calculation of operator product singularities on the example of the product $U\left(\beta_{1}\right) \chi \chi U\left(\beta_{2}\right)$ in the $\mathrm{O}(3)$ symmetric case. This product is given by (up to constant factors)

$$
\begin{aligned}
& \left(\beta_{21}+i \pi\right) \beta_{21} \int \frac{d \gamma_{1}}{2 \pi} \int \frac{d \gamma_{2}}{2 \pi}\left[\left(\gamma_{21}+i \pi\right) \gamma_{21} \frac{1}{\left(\gamma_{1}-\beta_{1}+i \pi\right)\left(\gamma_{1}-\beta_{1}\right)} \frac{1}{\left(\gamma_{2}-\beta_{1}+i \pi\right)\left(\gamma_{2}-\beta_{1}\right)} \times\right. \\
& \left.\frac{1}{\left(\beta_{2}-\gamma_{1}+i \pi\right)\left(\beta_{2}-\gamma_{1}\right)} \frac{1}{\left(\beta_{2}-\gamma_{2}+i \pi\right)\left(\beta_{2}-\gamma_{2}\right)}\right],
\end{aligned}
$$


with the contours specified by the prescription given in the main text. We have possible pinch singularities at $\beta_{2}=\beta_{1}$, $\beta_{2}=\beta_{1}-i \pi$ and $\beta_{2}=\beta_{1}-2 i \pi$. We can analyse their contribution by substituting $\beta_{1}=\beta_{2}+i \epsilon, \beta_{1}=\beta_{2}+i \pi+i \epsilon$ and $\beta_{1}=\beta_{2}+2 i \pi+i \epsilon$ respectively into the integral and studying the limit $\epsilon \rightarrow 0$. One finds a regular behaviour in the first two cases, which is caused by the zero of the preintegral factor, cancelling the pinch contribution. Only the third case will give a singular contribution, which will correspond to a first order pole in the operator product. Other operator products can be analysed similarly.

[1] Z. Horváth and G. Takács, Phys. Rev. D51 (1995) 2922-2932

[2] A. B. Zamolodchikov and Al. B. Zamolodchikov, Ann. Phys. (NY) 120 (1979) 165

[3] B. Berg, M. Karowski and P.Weisz, Phys. Rev. D19 (1979) 2477

[4] F. A. Smirnov, J. Phys. A17 (1984) L873

[5] F. A. Smirnov, J. Phys. A19 (1986) L575

[6] A. N. Kirillov and F. A. Smirnov, Phys. Lett. B198 (1987) 506

[7] F. A. Smirnov, Form-Factors in Completely Integrable Models of Quantum Field Theory, World Scientific (1992)

[8] D. Bernard and A. Leclair, Comm. Math. Phys. 142 (1991) 125

[9] F. A. Smirnov, Dynamical Symmetries of Massive Integrable Models I,II, RIMS preprints 772, 838 (1991)

[10] J.L. Cardy and G. Mussardo, Nucl. Phys. B340 (1990), 387

[11] A. Koubek and G. Mussardo, Phys. Lett. B311 (1993) 193

[12] F. A. Smirnov, Counting the Local Fields in SG Theory, preprint hep-th/9501059

[13] I. B. Frenkel and N. Yu. Reshetikhin, Comm. Math. Phys. 146 (1992) 1

[14] I. B. Frenkel and N. H. Jing, Proc. Nat'l. Acad. Sci. USA 85 (1988) 9373

[15] S. Lukyanov, S.L. Shatasvili, Phys. Lett. B298 (1993) 111-115

[16] S. Lukyanov, Comm. Math. Phys. 167 (1995) 183-226

[17] V. A. Fateev, E. Onofri and Al. B. Zamolodchikov, Nucl. Phys. B406 (1993) 421-565

[18] S. Lukyanov, Phys. Lett. B235 (1994) 409-417

[19] M. R. Niedermaier, On the Free Field Realization of Form Factors, preprint MPI-PhT/94-73, hep-th/9503139

[20] N. Reshetikhin and F. Smirnov, Comm. Math. Phys. 131 (1990) 157-178 